\title{
A ROBUST METHOD FOR QRS DETECTION BASED ON MODIFIED P-SPECTRUM
}

\author{
Peng Qiu ${ }^{1}$, and K. J. Ray Liu ${ }^{2}$ \\ ${ }^{1}$ Department of Radiology, Stanford University, Stanford, CA \\ ${ }^{2}$ Department of Electrical and Computer Engineering, University of Maryland, College Park, MD
}

\begin{abstract}
In this paper, we propose a robust method based on the modified p-spectrum to detect heart beats in ECG signals, which is also referred as QRS detection in the literature. QRS detection is an old problem that has been studied for several decades. In the literature, there are many methods based on various forms of transformations and thresholding, which require predetermined or fine-tuned thresholds. Another class of existing methods are based on machine learning, which require carefully labeled training data. In our study, we propose the modified $\mathrm{p}$-spectrum for QRS detection, which does not require either predetermined thresholds or training data, and can operate in real-time. The proposed method is evaluated using the MIT-BIH arrhythmia database.
\end{abstract}

Index Terms - ECG, QRS detection

\section{INTRODUCTION}

Electrocardiograph (ECG) is the electrical voltage in the heart. The ECG signal contains a lot of useful information for diagnosis, i.e. heart rate, heart rate variability, etc. The extraction of this information usually relies on the accurate detection of heart beats, which is also referred as QRS detection in the literature. The problem of QRS detection has been studied for several decades. A review of existing methods can be found in [1].

Existing QRS detection methods can be mainly divided into two categories. One category is based on various forms of transformations and thresholding, such as derivatives and digital filters, wavelet transform, match filter, mathematical morphology, etc. These methods either require predetermined thresholds [2], or determine the thresholds adaptively [3]. The predetermined thresholds and adaptation rules are usually patient dependent, i.e. the statistics of a patient's ECG signal, shape of the waveform of the QRS complex, sampling frequency, etc. The other category is based on machine learning, such as neural network, hidden markov model, etc. The machine learning methods require carefully labeled training data, which is also patient dependent [4].

In this study, the motivation is to develop a robust QRS detection method that is not patient dependent. A modified $\mathrm{p}$-spectrum is proposed base on the $\mathrm{p}$-spectrum in [5]. The $\mathrm{p}$ - spectrum, also known as the periodicity spectrum, is a robust method for periodicity detection. It has several advantages over the Fourier transform. Since the p-spectrum is a transform based on the data singularity, the p-spectrum of a periodic signal will have high sharp peaks. Also, the p-spectrum is not affect by the shape of waveform within each period, and can tolerate amplitude scaling across different periods. On the other hand, the disadvantage is that, the p-spectrum is sensitive to phase shift. In ECG signals, the time interval between consecutive heart beats is not a constant, because of the heart rate variability. And thus, the ECG signal resembles a signal with phase shift. Therefore, a direct application of the p-spectrum in QRS detection does not yield satisfying performance.

In this paper, we propose a modified p-spectrum to address the issue of phase shift. Since the modified p-spectrum is a form of transformation, we do not require any training data. By inheriting the advantages of p-spectrum, i.e., high sharp peaks, the proposed method does not require any predetermined thresholds, either. The only assumption is the similarity between the waveforms of consecutive heart beats. Therefore, the proposed method is not patient dependent, and can be applied to different datasets without training or finetuning. Moreover, the proposed method can operate in realtime. The rest of this paper is organized as follows. We first give a brief review of the $\mathrm{p}$-spectrum, and propose the modified p-spectrum. The proposed method for QRS detection is described in detail using an example. In the Results section, we evaluate the detection performance using the MITBIH database.

\section{METHOD}

\subsection{The p-spectrum}

Given a discrete time signal or the sampled version of a continuous time signal, $\left\{x_{1}, x_{2}, \ldots, x_{N}\right\}$, the p-spectrum is calculated as follows. Let integer $p$ be the possible value of the period. The signal is rearranged into the following:

$A_{p}=\left(\begin{array}{ccccccc}x_{1} & x_{2} & \cdots & x_{i} & x_{i+1} & \cdots & x_{p} \\ x_{p+1} & x_{p+2} & \cdots & x_{p+i} & x_{p+i+1} & \cdots & x_{2 p} \\ \cdots & \cdots & \cdots & \cdots & \cdots & \cdots & \cdots \\ x_{m p+1} & x_{m p+2} & \cdots & x_{N} & 0 & \cdots & 0\end{array}\right)$ 
where the signal is broken into non-overlapping segments of length $p$, and the non-overlapping segments form the rows of the matrix $A_{p}$. Since the total length of the signal $N$ may not be a multiple of $p$, zeros are used to fill the empty entries in the last row, or the last row is discarded. Singular value decomposition (SVD) is performed obtain the singular values of $A_{p}$. The ratio between the largest and the second largest singular values is defined as the amplitude that corresponds to the "frequency" $p$. By varying the value of $p$, the ratio is a function of $p$, which is the $\mathrm{p}$-spectrum.

The p-spectrum detects periodicity based on the singularity of the matrix $A_{p}$. For example, Let $\left\{x_{i}\right\}$ be a periodic signal of period $p_{0}$. If the value of $p$ equals to the period $p_{0}$, then $A_{p}$ will have identical rows (except the last row), and the second largest singular value will be close to zero. Therefore, the amplitude that corresponds to "frequency" $p=p_{0}$ goes to infinity. When $p$ equals to multiples of $p_{0}$, the amplitude of the p-spectrum also goes to infinity. When $p$ equals to other values, the rows of $A_{p}$ are shifted versions of each other, with no dominating component, and the amplitude of the p-spectrum tends to be of a moderate value. Therefore, the peaks in the $\mathrm{p}$-spectrum are indicators of the periodicity. The p-spectrum is not affect by the shape of waveform within each period, and can tolerate amplitude scaling across different periods. However, the p-spectrum is sensitive to phase shift. Because with the presence of phase shift, the rows of the matrix $A_{p}$ will never be perfectly aligned, resulting in a p-spectrum without significant peaks.

\subsection{Modified p-spectrum}

In ECG signals, because of the heart rate variability, the time interval between consecutive heart beats is not a constant. The ECG signal resembles a signal with phase shift. Thus, a direct application of the p-spectrum for QRS detection does not yield satisfying performance (an example is shown in the following subsection). In this work, we propose a modified p-spectrum to solve the problem of QRS detection in ECG signals.

Given the sampled version of an ECG signal, $\left\{x_{1}, \ldots, x_{N}\right\}$, the modified $p$-spectrum is defined as follows. Let $p$ be an integer. The signal is rearranged into the following matrix:

$$
A_{p}^{\prime}=\left(\begin{array}{cccc}
x_{1} & x_{2} & \cdots & x_{p} \\
x_{p+1} & x_{p+2} & \cdots & x_{2 p}
\end{array}\right)
$$

where the signal is broken into non-overlapping segments of length $p$, and the first two non-overlapping segments form the rows of the matrix $A_{p}^{\prime}$. The ratio between the larger and the smaller singular values of $A_{p}^{\prime}$ is defined as the amplitude that corresponds to the "frequency" $p$. By varying the value of $p$, the ratio is a function of $p$, which is the modified $\mathrm{p}$ spectrum. The modified $\mathrm{p}$-spectrum inherits the advantages of the p-spectrum. Therefore, the modified $p$-spectrum is also not affect by the shape of waveform within each period, and can tolerate amplitude scaling across different periods.

The modified p-spectrum is able to detect heart beat from ECG signals with heart rate variability. For example, let $T_{1}$ be the interval between the first two heart beats, and let $T_{2}$ be the intervals between the second to the fifth consecutive heart beats. Because of the heart rate variability, there is moderate difference between the values of $T_{1}, T_{2}$. Denote $f_{s}$ as the sampling frequency. When $p=T_{1} f_{s}$, the first $p$ points constitute the first heart beat period, and the following $p$ points constitute the second heart beat period. Under the assumption that the waveforms of ECG signals in consecutive heart beats are similar, the two rows of $A_{p}^{\prime}$ are similar, and the ratio between the two singular values is large. Therefore, in the modified p-spectrum, we are able to observe a peak at $p=T_{1} f_{s}$. The only exception is that when $T_{2}$ is very small, when $p=T_{1} f_{s}$, the second row may contain both the second and the third peaks. In this case, we will not observe high peak at $p=T_{1} f_{s}$, and a detection error may occur.

Since the modified p-spectrum is obtained based on the singularity of $A_{p}^{\prime}$, as long as the consecutive heart beats share similar waveform, the peak at $p=T_{1} f_{s}$ is sharp and distinguishing, which can be identified without predetermined threshold. When the value of $T_{1} f_{s}$ is identified, we know that the first heart beat happened between time 0 and time $T_{1}$, and the second heart beat happened between time $T_{1}$ and time $2 T_{1}$. Therefore, the maximum amplitude of the ECG signal between 0 and $T_{1}$ indicates the first heart beat; and the maximum amplitude of the ECG signal between $T_{1}$ and $2 T_{1}$ indicates the second heart beat.

In the modified p-spectrum, the beginning portion of the ECG signal is examined to detect the first and second heart beats. Then, we discard the data that corresponds to the first heart beat, and apply the same analysis to identify the first two heart beats in the remaining signal, which are the second and third heart beats in the original ECG signal. In this way, the modified p-spectrum can operate in real time. In the following subsection, we use an example to show how the modified pspectrum is applied for QRS detection.

\subsection{QRS detection}

In this subsection, we describe the details of the proposed QRS detection method using an example. The example ECG signal is acquired at a sampling frequency of $f_{s}=200 \mathrm{~Hz}$. The beginning part of the ECG signal is shown in Figure 1. Although it is not visually obvious, the intervals between consecutive heart beats are different. As mentioned above, due to the heart rate variability, a direct application of the $p$-spectrum does not reveal the periodicity. The p-spectrum of this example is shown in Figure 2, where the unit of the horizontal axis is second $\left(p / f_{s}\right)$, and no distinguishing peak is observed.

As discussed in the previous subsection, in the modified $\mathrm{p}$ spectrum, we expect to see a peak, where the value of $p$ corre- 


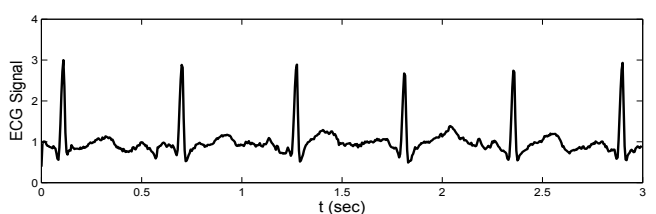

Fig. 1. Example of ECG signal.

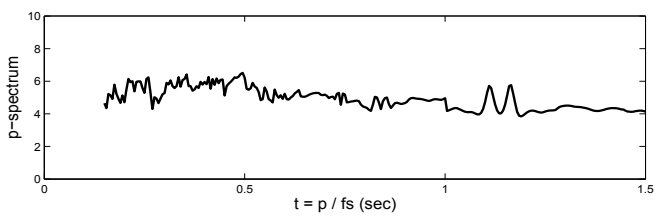

Fig. 2. P-spectrum of the example ECG signal.

sponds to the interval between the first and second heart beat $T_{1}$. Therefore, when examining the modified $\mathrm{p}$-spectrum, we should choose the range of $p$ to cover the value that corresponds to $T_{1}$. We assume that the maximum heart rate is 400 beats per minute, and the minimum heart rate is 40 beats per minute. Under such assumption, the value of $T_{1}$ can vary between $0.15 \mathrm{sec}$ and $1.5 \mathrm{sec}$. Since the sampling frequency in this example is $200 \mathrm{~Hz}$, we should examine the value of $p$ in the following range, $[30,300]$. In Figure 3 (a), the modified p-spectrum is shown, with $p$ varying from 30 to 300 . The horizontal axis is $p / f_{s}$. In Figure 3 (b) (c) (d) (e), we show the first and second rows of the $A_{p}^{\prime}$ matrices that correspond to the cases indicated by the arrows in Figure 3 (a).

From these figures,we can see that: for case (b), $p=40$ which corresponds to $0.195 \mathrm{sec}$, since the two rows of $A_{p}^{\prime}$ are not similar, the ratio between the two singular values is small. For case (c) $p=118$, corresponding to $0.59 \mathrm{sec}$, there is a sharp peak in the modified p-spectrum, indicating that the interval between the first two heart beats is $0.59 \mathrm{sec}$. In cases (d) and (e), the value of $p$ is large such that the first row of $A_{p}^{\prime}$ contains the first two heart beats, and the second row of $A_{p}^{\prime}$ contains the third and fourth heart beats. In these cases, due to the heart rate variability, the two rows of $A_{p}^{\prime}$ can not be perfectly aligned, and two small peaks are observed in the modified p-spectrum. For an ECG signal that contains very small heart rate variability, the two small peaks may merge into one sharp peak, which corresponds to two times the interval between the first two heart beats. By detecting peaks in the modified p-spectrum, we are able to identify the time interval between the first two heart beats, $T_{1}$.

We notice that, although not shown in this example, for some of the ECG data we examined, the modified p-spectrum contains a sharp peak at the $p$ value that corresponds to case (b) in Figure 3, which complicates the identification of the value of $T_{1}$. This is because of the structure of the p-wave in the ECG signal. To suppress this undesired peak, we propose an auxiliary spectrum, which is defined as follows. The horizontal axis of the auxiliary spectrum is exactly the same

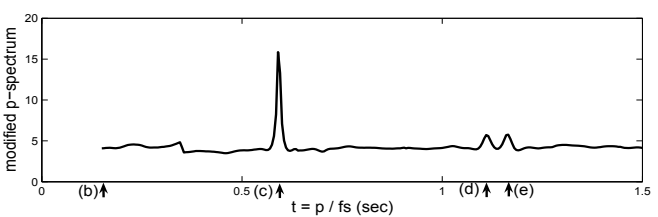

(a)
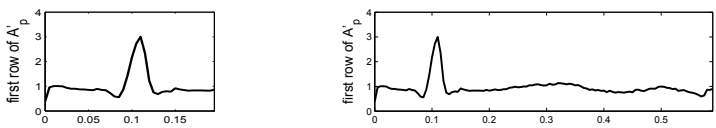

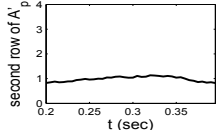

(b)

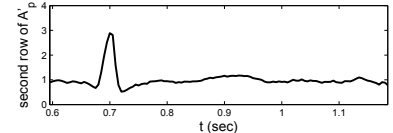

(c)
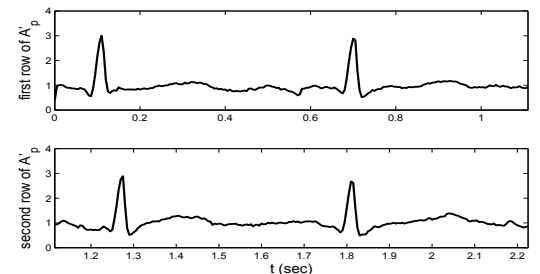

(d)
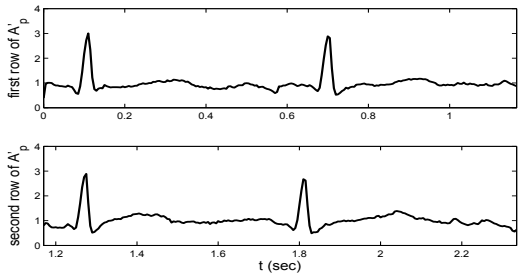

(e)

Fig. 3. The modified p-spectrum is shown in Fig (a). Fig (b) (c) (d) (e) show the first and second rows of the $A_{p}^{\prime}$ matrices that correspond to the arrows in Fig (a).

with that of the modified p-spectrum. The only difference is how the amplitude of the auxiliary spectrum is defined. In the auxiliary spectrum, for a particular value $p$, the $A_{p}^{\prime}$ matrix is obtained using (2). The amplitude of the auxiliary spectrum is defined as the maximum of the sum of the two rows of $A_{p}^{\prime}$.

The auxiliary spectrum of the example ECG signal (Figure 1) is shown in Figure 4. The auxiliary spectrum takes into account of the amplitude information of the ECG signal. For case (b) in Figure 3 or other cases when the heart beats in the two rows of $A_{p}^{\prime}$ are not perfectly aligned, the amplitude of the auxiliary spectrum is about half of the maximum amplitude of the ECG signal. For cases (c) (d) and (e) in Figure 3 , there is at least one heart beat perfectly aligned in the two rows of $A_{p}^{\prime}$, making the amplitude of the auxiliary spectrum to be comparable with the maximum amplitude of the ECG signal. Compared with the modified $\mathrm{p}$-spectrum, the auxiliary spectrum does not have the undesired peak mentioned above. 


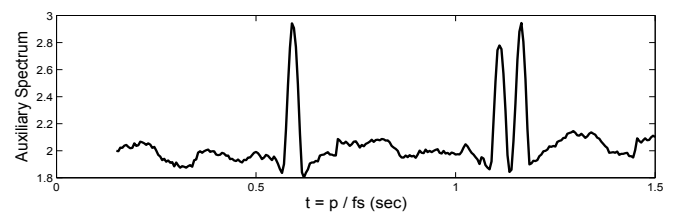

Fig. 4. The auxiliary spectrum of the example ECG signal.

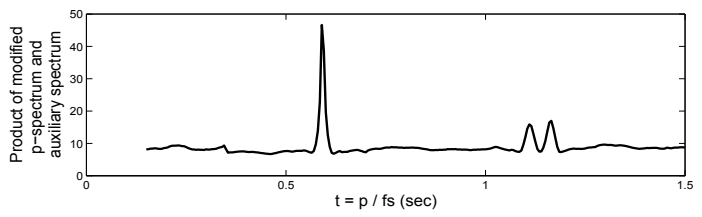

Fig. 5. The product of the modified p-spectrum and the auxiliary spectrum.

However, the disadvantage of the auxiliary spectrum is, the peaks are much wider and smoother, with a contrast of two fold, while the modified p-spectrum can provide sharp peaks.

Taking advantage of both the modified p-spectrum and the auxiliary spectrum, we proposed to identify the value of $T_{1}$ based on their product in Figure 5, where the undesired peak mentioned above is suppressed, and the sharpness of peaks in the modified p-spectrum is preserved. The product of the modified p-spectrum and the auxiliary spectrum contains only the peak that correspond to $T_{1}$, and peaks that correspond to multiples of $T_{1}$ (if exist). Using a sliding window, it is easy to identify the value of $T_{1}$. After the value of $T_{1}$ is determined, the times of the first two heart beats can be detected as follows. The time point that corresponds to the maximum amplitude of the ECG signal between 0 and $T_{1}$ indicates the first heart beat; while the time point that corresponds to the maximum amplitude of the ECG signal between $T_{1}$ and $2 T_{1}$ indicates the second heart beat. In this example, $T_{1}$ is identified to be $0.59 \mathrm{sec}$, and the first two heart beats are correctly identified.

After the detection of the first two heart beats, we remember the time point that corresponds to the first heart beat, throw away the ECG signal from the beginning to a short time interval before the second heart beat, forget about the detection result for the second heart beat, regard the remain data as a new problem, and detect the first two heart beats in the remaining data. Then, the process iterates. When throwing away the beginning part of the data, the length of the short time interval does not affect the detection performance, as long as the segment of the first QRS complex is discarded. This is because, for every iteration, the remaining data is regarded as a new problem of detecting the first two heart beats. Also, when there is detection error in one iteration, the error does not propagate, because of the same reason. Note that only a beginning portion of the ECG signal is needed to detect the first two heart beats, therefore, the proposed method can operate in real time with a small amount of delay.

\section{RESULTS}

We evaluate the modified p-spectrum method using the MIT$\mathrm{BIH}$ database [6], which contains 48 ECG data of half hour recording. The first channel of the ECG data is examined. The detection performance is evaluated by the sensitivity $(S e=$ $\left.\frac{T P}{T P+F N}\right)$ and positive predictivity $\left(+P=\frac{T P}{T P+F P}\right)$, where $T P$ is the number of correctly detected heart beats, $F N$ is the number of missed detection, and $F P$ is the number of false positive detection. The average performance over all 48 datasets is $S e=94.32 \%$ and $+P=97.66 \%$.

\section{CONCLUSION}

The best QRS detection performances reported so far is about $99 \%$ [1], which are based on either thresholding or machine learning. However, the existing methods either require predetermined or fine-tuned thresholds, or require carefully labeled training data. When such data-dependent specification is not accurate enough, the performance will degrade significantly. Therefore, the achievable detection accuracy comes at the cost of dependence of patient and scenario. On the other hand, the proposed modified p-spectrum is much more robust, in the sense that there is no need to determine any parameters for different datasets. Moreover, the average detection accuracy is about $96 \%$, which is close to the best thresholding or training based detection performance reported in the literature.

\section{REFERENCES}

[1] B-U. Kohler, C. Hennig, and R. Orglmeister, "The Principles of Software QRS Detection", IEEE Engineering in Med. and Biol., Jan.-Feb. 4257, 2002.

[2] G.M. Friesen, T.C. Jannett, M.A. Jadallah, S.L. Yates, S.R. Quint, and H.T. Nagle, "A comparison of the noise sensitivity of nine QRS detection algorithms", IEEE Trans on Biomed Eng, 37:85-98, 1990.

[3] I.I. Christov, "Real time electrocardiogram QRS detection using combined adaptive threshold", Biomed Eng Online, 3(1):28, 2004.

[4] P. Ravier, and O. Buttelli, "Robust detection of qrs complex using klauder wavelets", European Signal Processing Conference, 2199-2202, 2004.

[5] P.P. Kanjilal, J. Bhattacharya, and G. Saha, "Robust method for periodicity detection and characterization of irregular cyclical series in terms of embedded periodic components", Phys. Rev. E, 59:4013-4025, 1999.

[6] R. Mark, and G. Moody, "MIT-BIH Arrhythmia data base directory", Cambridge: Massachusetts Institute of Technology, 1988. 